\title{
The effects of family structure and function on mental health during China's transition: a cross-sectional analysis
}

\author{
Yao Cheng, Liuyi Zhang, Fang Wang, Ping Zhang, Beizhu Ye and Yuan Liang*
}

\begin{abstract}
Background: Social change, intensified by industrialization and globalization, has not only changed people's work lives but also their personal lives, especially in developing countries. The aim of this study was to provide evidence and recommendations regarding family structure, function, and mental health to actively respond to rapid social change.
\end{abstract}

Methods: A cross-sectional survey was conducted face-to-face and door-to-door from July 2011 to September 2012 in Hubei Province, central China. Family structure comprised alone, couple, nuclear family, and extended family; family function was measured using the family APGAR (Adaptation, Partnership, Growth, Affection, and Resolve) scale, and mental health was measured using the Chinese version of the 12-item General Health Questionnaire (GHQ-12).

Results: The urban-vs-rural difference of family structure among alone, couple, nuclear family, and extended family was statistically significant (5.21\% vs $4.62 \%$; $27.36 \%$ vs $13.14 \% ; 33.22 \%$ vs $27.74 \% ; 34.20 \%$ vs $54.50 \%$, respectively; $p<0.0001$ ); and those difference of family function was not statistically significant (8.11 \pm 2.13 vs $8.09 \pm 2.27, p=0.9372$ ). The general linear regression showed that the effect of family structure on mental health, whether urban or rural, was not significant, however, the effect of family function was significant, especially regarding better family functioning with better mental health.

Conclusions: Combined the effects of family structure and function on mental health, the external form of family (family structure) may not be important; while the internal quality of role (family function) might be key. Improving the residents' family function would be a priority strategy for family practice with their mental health.

Keywords: Mental health, Family structure, Family function, Urban-rural difference

\section{Background}

Social change, intensified by industrialization and globalization, has not only changed people's work lives but also their personal lives, especially in developing countries [1]. Since the reform and opening-up of China in 1978, and especially in recent decades, Chinese society has undergone unprecedented changes. In the 1982 Chinese Census, the urban population accounted for $20.02 \%$ of the total population, increasing to $36.09 \%$ by 2000 and to

\footnotetext{
*Correspondence: liangyuan217@hust.edu.cn

Department of Social Medicine and Health Management, School of Public

Health, Tongji Medical College, Huazhong University of Science and

Technology, Wuhan, China
}

$49.68 \%$ in the 2010 Census [2]. Although the growth of the urban population has brought about rapid economic development, it has also resulted in increased pressure on employment, transportation, housing, education, and public health, especially mental health [3]. At the same time, a rapid decline in the rural population combined with the widening urban-rural gap has not only affected agricultural labor, but also the lives of farmers, including their mental health [4]. Another change has been observed in the family structure. This fact is depicted in the reduction of the number of the traditional extended and nuclear families and the increase in the number of alone and couple families [5-7]. 
Although there are many studies regarding family structure and function and mental health, most focus on special groups such as children, adolescents, immigrants, etc., with very few looking at adult populations [8-12]. However, the family is the basic social unit of the general population and may have an important effect on mental health at all ages. Furthermore, because of intense competition in industrialization and globalization, adult populations face greater pressure in their work and personal lives, and more adults are likely to suffer from mental disorders [13]. Additionally, conflicts between culture and values arising from globalization can not only affect adults' employment, but also their family life and mental health $[14,15]$. Therefore, a study on the adult population's family structure and function as well as their mental health has important practical significance, especially for developing countries that currently hold a disadvantaged position in global competition. With the world's largest population, China's various social problems stemming from industrialization and globalization are very complex. Therefore, China's experience, whether as a success or failure, may provide important reference values for other developing countries.

To address the above problems, the current study provides an empirical analysis of the effects of family structure and function on mental health via a population-based survey in Hubei Province, central China. The aim of this study is to provide evidence and recommendations regarding family structure, function, and mental health to actively respond to rapid social change.

\section{Methods}

\section{Participants}

Hubei province, where the provincial GDP ranking has been moderate from 2011 to 2014 in China, was determined as the source site of sample. Three cities, Wuhan, Xiaogan and Qianjiang, were selected using purposive sampling, represented respectively of provincial capital city, prefecture-level city and county-level city in Hubei Province. According to Hubei Provincial Bureau of Statistics, the total population (including urban and rural residents) of Wuhan, Xiaogan and Qianjiang city is 8.29, 5.31 and 1.03 million, respectively. In general, urban residents accounted for $54.51 \%$ in Hubei province.

The current survey was conducted face-to-face and door-to-door from July 2011 to September 2012. The sample size in this study was calculated to be no fewer than 576 individuals by the following determination formulas: $n=\left(t^{2} \times \pi^{2} / \delta^{2}\right) \times$ deff (design effect, 1.5), $t=1.96$, $\pi=0.5, \delta=5 \%, n \approx 576$, and the total of urban and rural sample is not less than 1152 cases. A method of stratified cluster $\mathrm{r}$ sampling was adopted to acquire the study sample. Two communities in urban areas and two administrative villages in rural areas were selected from each city using convenience sampling. The adult residents aged 15 years and older in the selected communities and villages were our targeted samples. Inclusion criteria were the residents aged 15 years and older and willing to participate in the study. Excluded were the residents suffered from terminal illness, diagnosed mental disorder, and not willing to participate. Figure 1 shows the flowchart for recruitment and response rates. Excluding questionnaires with missing data, a total of 1052 (65.96\%) valid questionnaires were used in the study.

\section{Measures \\ Independent variables}

Family structure operationalized on the basis of current living arrangement, and comprised the following categories: alone, couple, nuclear family, and extended family. Specifically, alone means a single-person household, and couple means a household with just one couple. It is worth noting the definition of the nuclear family in the current study is used in Chinese way, namely parents living with unmarried children or single parent living with unmarried children, regardless of the age of unmarried children $[2,16]$. This definition is in line with the actual situation of Chinese family and cultural traditions. In China, children, even adult children, as long as not married, the vast majority of them are living with their parents. In addition, Chinese extended family means older parents living with their married children and possibly other relatives (representing a traditional Chinese family structure where three or four generations live under the same roof).

Family function was measured using the family APGAR scale, developed by Smilkstein to assess family function on five aspects (Adaptation, Partnership, Growth, Affection, and Resolution) with a three-point scale ranging from 0 (hardly ever) to 2 (almost always), and it is suitable for use in any ages [17]. The Chinese version of the APGAR was still reliable and repeatable $[18,19]$, and had relatively good internal consistency (a $=0.75$ ) in the current study. The final family function score is the sum of all five aspects with higher APGAR scores denoting better family function. According to previous studies, an APGAR score of $0-3$ is considered to be poor, $4-6$ is fair, and $7-10$ is good $[20,21]$.

\section{Dependent variable and control variables}

The General Health Questionnaire (GHQ) is one of the most common mental health tools developed by D.P. Goldberg in the 1970s [22-24]. We used GHQ-12 to measure individuals' general psychological healthy status. The Chinese version GHQ-12 was still reliable and repeatable and had relatively good internal consistency $(a=0.76)$ in the current study. The questionnaire consists of 12 items assessing the severity of a mental 


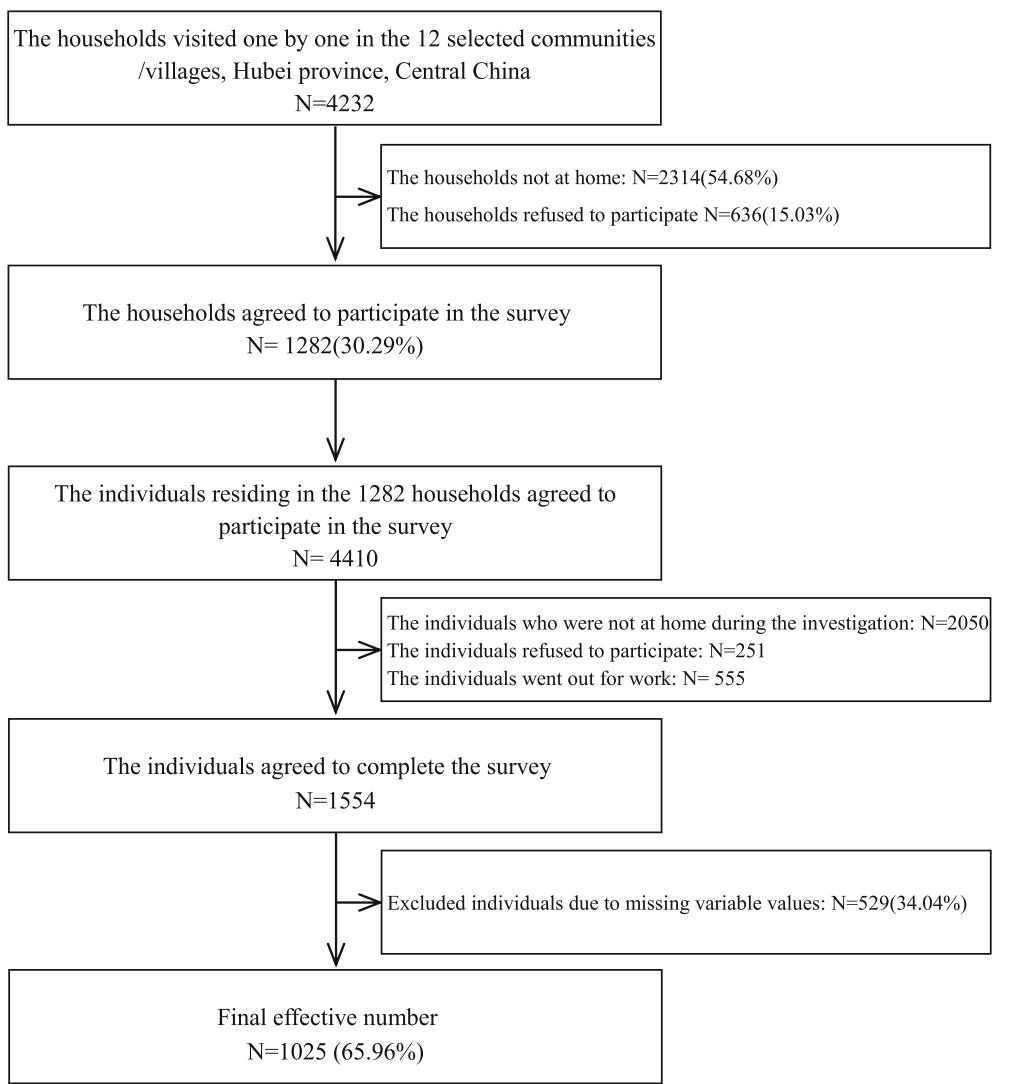

Fig. 1 Flowchart for recruitment and response rates to the survey

problem using a 4-point scale from 0 (never) to 3 (always). Following previous studies, we used a bimodal dataset (0-0-1-1) for scoring that participates indicated low tendency of mental health were defined as 0 , or else they were defined as 1 . Thus, the possible score range is $0-12$. GHQ-12 scores of four and above indicate tendency of mental disorder [25-27]. Sociodemographic variables (i.e., gender, age, education and marital status), self-reported family economic status, smoking, drinking and chronic disease status (e.g., self-reported assessment of a doctor-diagnosed hypertension, diabetes, chronic obstructive pulmonary disease, asthma, and arthritis) were used as control variables.

\section{Statistical analysis}

A descriptive analysis was carried out for the primary variables. The differences in the primary variables between urban and rural residents were tested using Pearson's $\chi^{2}$ tests. ANOVA analysis was used to compare GHQ-12 scores and APGAR scores among different family structure, and Bonferroni post-hoc tests were performed. Three generalized linear models were used to examine the independent effects of family structure and function on GHQ-12. Model 1 includes family structure and function only. Gender, age, education, marital status, and self-reported family economic status were added to Model 2, and smoking, drinking, and chronic disease status were added to Model 3.

\section{Results}

Table 1 shows the distribution characteristics of the research variables. There were fewer male participants than female ( $40.59 \%$ vs $59.41 \%)$, perhaps because more males were out at work when residents were approached to take part in the survey. Participants aged 60 years or older represented the largest proportion of respondents (41.37\%), perhaps again because younger participants were out at work when residents were asked to participate. The proportion of chronic disease status (48.00\%) might be related to the proportion of older adults ( $\geq 60$ years old) of the participants.

The urban-vs-rural difference of family structure among alone, couple, nuclear family, and extended family was statistically significant $(5.21 \%$ vs $4.62 \% ; 27.36 \%$ vs $13.14 \%$; $33.22 \%$ vs $27.74 \%$; $34.20 \%$ vs $54.50 \%$, respectively; $p<0.0001)$. Family function was generally good $(8.08 \pm 2.18)$, and the urban-vs-rural difference was not statistically significant $(8.11 \pm 2.13 \mathrm{vs} \quad 8.09 \pm 2.27 \quad p=$ $0.9372)$. GHQ-12 scores were generally low $(2.92 \pm 2.64)$, indicating some serious degree of mental illness [], and 
Table 1 Descriptive Statistics for the primary variables $(n=1025)$

\begin{tabular}{|c|c|c|c|c|c|c|c|c|}
\hline \multirow[t]{2}{*}{ Variables } & \multicolumn{2}{|l|}{ Total } & \multicolumn{2}{|c|}{$\operatorname{Urban}(n=614)$} & \multicolumn{2}{|c|}{$\operatorname{Rural}(n=411)$} & \multirow[t]{2}{*}{$x^{2} / F$} & \multirow[t]{2}{*}{$P$} \\
\hline & N/Mean & $\% / S D$ & N/Mean & $\% / S D$ & N/Mean & $\% / S D$ & & \\
\hline \multicolumn{9}{|l|}{ Sex } \\
\hline Male & 416 & 40.59 & 250 & 40.72 & 166 & 40.39 & \multirow[t]{2}{*}{0.0109} & \multirow[t]{2}{*}{0.9167} \\
\hline Female & 609 & 59.41 & 364 & 59.28 & 245 & 59.61 & & \\
\hline \multicolumn{9}{|l|}{ Age (years old) } \\
\hline $15-29$ & 117 & 11.41 & 69 & 11.24 & 48 & 11.68 & \multirow[t]{5}{*}{4.8173} & \multirow[t]{5}{*}{0.3066} \\
\hline $30-39$ & 115 & 11.22 & 73 & 11.89 & 42 & 10.22 & & \\
\hline $40-49$ & 171 & 16.68 & 100 & 16.29 & 71 & 17.27 & & \\
\hline $50-59$ & 198 & 19.32 & 107 & 17.43 & 91 & 22.14 & & \\
\hline$\geq 60$ & 424 & 41.37 & 265 & 43.16 & 159 & 38.69 & & \\
\hline \multicolumn{9}{|l|}{ Ethnicity } \\
\hline Han nationality & 1016 & 99.12 & 606 & 98.70 & 410 & 99.76 & \multirow[t]{2}{*}{3.1730} & \multirow[t]{2}{*}{0.0749} \\
\hline Others & 9 & 0.88 & 8 & 1.30 & 1 & 0.24 & & \\
\hline \multicolumn{9}{|l|}{ Religion } \\
\hline Yes & 36 & 3.51 & 27 & 4.40 & 9 & 2.19 & \multirow[t]{2}{*}{3.5372} & \multirow[t]{2}{*}{0.0600} \\
\hline No & 989 & 96.49 & 587 & 95.60 & 402 & 97.81 & & \\
\hline \multicolumn{9}{|l|}{ Education } \\
\hline College or more & 104 & 10.15 & 98 & 15.96 & 6 & 1.46 & \multirow[t]{4}{*}{191.1864} & \multirow[t]{4}{*}{$<.0001$} \\
\hline Senior high & 244 & 23.80 & 204 & 33.22 & 40 & 9.73 & & \\
\hline Junior high & 305 & 29.76 & 175 & 28.50 & 130 & 31.63 & & \\
\hline Primary or less & 372 & 36.29 & 137 & 22.31 & 235 & 57.18 & & \\
\hline \multicolumn{9}{|l|}{ Self-reported economic status } \\
\hline Good & 154 & 15.02 & 96 & 15.64 & 58 & 14.11 & \multirow[t]{3}{*}{59.3292} & $<.0001$ \\
\hline Fair & 635 & 61.95 & 427 & 69.54 & 208 & 50.61 & & \\
\hline Bad & 236 & 23.02 & 91 & 14.82 & 145 & 35.28 & & \\
\hline Smoking & & & & & & & & \\
\hline Yes & 217 & 21.17 & 117 & 19.06 & 100 & 24.33 & 4.1018 & 0.0428 \\
\hline No & 808 & 78.83 & 497 & 80.94 & 311 & 75.67 & & \\
\hline Drinking & & & & & & & & \\
\hline Yes & 153 & 14.93 & 81 & 13.19 & 72 & 17.52 & 3.6248 & 0.0569 \\
\hline No & 872 & 85.07 & 533 & 86.81 & 339 & 82.48 & & \\
\hline Chronic disease status & & & & & & & & \\
\hline Yes & 492 & 48.00 & 297 & 48.37 & 195 & 47.45 & 0.0845 & 0.7713 \\
\hline No & 533 & 52.00 & 317 & 51.63 & 216 & 52.55 & & \\
\hline Family structure & & & & & & & & \\
\hline Alone & 51 & 4.98 & 32 & 5.21 & 19 & 4.62 & 49.4675 & $<.0001$ \\
\hline Couple & 222 & 21.66 & 168 & 27.36 & 54 & 13.14 & & \\
\hline Nuclear family & 318 & 31.02 & 204 & 33.22 & 114 & 27.74 & & \\
\hline Extended family & 434 & 42.34 & 210 & 34.20 & 224 & 54.50 & & \\
\hline Family function & & & & & & & & \\
\hline (Total APGAR score) & 8.10 & 2.18 & 8.11 & 2.13 & 8.09 & 2.27 & 0.01 & 0.9372 \\
\hline Levels of family function & & & & & & & & \\
\hline Good (APGAR score $=7-10$ ) & 810 & 79.02 & 487 & 79.32 & 323 & 78.59 & 0.1951 & 0.9071 \\
\hline
\end{tabular}


Table 1 Descriptive Statistics for the primary variables ( $n=1025)$ (Continued)

\begin{tabular}{llllllll}
\hline Fair (APGAR score $=4-6)$ & 171 & 16.68 & 102 & 16.61 & 69 & 16.79 & \\
$\quad \begin{array}{l}\text { Bad (APGAR score }=0-3) \\
\text { Mental health }\end{array}$ & 44 & 4.29 & 25 & 4.07 & 19 & 4.62 & \\
$\quad($ GHQ-12 score) & 2.92 & 2.64 & 2.44 & 2.34 & 3.65 & 2.89 & 54.81 \\
$\begin{array}{l}\text { Tendency of mental disorder } \\
\text { Yes (GHQ-12 score } \geq 4)\end{array}$ & 354 & 34.54 & 161 & 26.22 & 193 & 46.96 & 46.7824 \\
No (GHQ-12 score $<4)$ & 671 & 65.46 & 453 & 73.78 & 218 & 53.04 & $<.0001$ \\
\hline
\end{tabular}

Note: Family function was measured by APGAR scale; mental health was measured by GHQ-12. Total APGAR scores of family function and GHQ-12 scores of mental health between urban and rural populations were compared by ANOVA analysis

the urban-vs-rural difference was statistically significant $(2.44 \pm 2.34$ vs $3.65 \pm 2.89 ; p<0.0001)$. The prevalence of the tendency of mental disorder of rural residents is higher than those of urban residents)(26.22\% vs $46.96 \%$; $p<0.0001$ ).

Table 2 shows the rural and urban differences between family function and family structure. For urban participants, the family structure "couple" obtained the best function score $(8.54 \pm 1.88)$, and "alone" (7.69 \pm 2.71$)$ obtained the lowest $(F=3.88 ; p=0.0092)$. Interestingly, for rural participants, the highest score for family functioning was for those living alone $(8.95 \pm 1.61)$, and the lowest was for couples $(7.63 \pm 2.62)$. The differences between family function and family structure was not statistically significant $(F=1.68 ; p=0.1712)$.

Table 3 shows the results of the general linear regression for the effect of the independent variables on mental health (GHQ-12). Model 1 (with family structure and function alone) showed, for both urban and rural residents, that the effect of family structure was not significant. However, the effect of family function was significant, especially regarding better family functioning with better mental health. Similar results were obtained for Models 2 and 3 (adding sociodemographic variables and then smoking, alcohol and chronic disease, respectively). Furthermore, there was little change in $\beta$ values between Models 2 and 3.

\section{Discussion}

This study provides an evidence for the effects of family structure and function on mental health of adult

Table 2 The association of family structure and family function (APGAR)

\begin{tabular}{|c|c|c|c|c|c|c|c|c|}
\hline \multirow[t]{2}{*}{ Family structure } & \multicolumn{4}{|l|}{ Urban } & \multicolumn{4}{|l|}{ Rural } \\
\hline & Mean & SD & $F$ & $P$ & Mean & SD & $F$ & P \\
\hline Alone & 7.69 & 2.71 & 3.88 & 0.0092 & 8.95 & 1.61 & 1.68 & 0.1712 \\
\hline Couple & 8.54 & 1.88 & & & 7.63 & 2.62 & & \\
\hline Nuclear family & 7.84 & 2.10 & & & 8.10 & 2.35 & & \\
\hline Extended family & 8.08 & 2.19 & & & 8.13 & 2.18 & & \\
\hline
\end{tabular}

Note: Family structure with urban/rural setting, $F=4.43 ; P=0.0042$ population during China's transition. Overall, our findings suggest that the urban-rural difference of family structure was statistically significant, and those of family function was not statistically significant; and the effect of family structure on mental health, whether urban or rural, was not significant, however, those of family function was significant.

\section{Family structure and function}

The change in family structure may be related to two aspects. One concerns an internal cause; that is, familial changes brought about for personal reasons. For example, older family members (especially older parents) may choose to live alone because they do not want to burden their children or grandchildren [28]. Meanwhile, younger family members (especially adult children living in the city) cannot afford to preserve the traditional extended family lifestyle, and may not have the ability to support their parents (because of rising house prices, job instability, and a lack of income security) [29, 30]. A further reason, external to the family situation, is conflict between culture and values. The past 20-30 years, with the advent of globalization and fierce market competition, have marked the rise of the ego and money worship, with a decrease in altruism and spiritualism [31]. Such changes will not only affect people's work and family lives, but also create interpersonal tensions and work-family conflict, as well as impacting on mental health [32].

Because of the difference in measurement tools, evaluation methods and participants, comparative analysis of family function is difficult among different studies. In the current study, overall, family function is good (the proportion deemed "poor" only accounted for $3.85 \%$ in urban areas and $4.67 \%$ in rural areas, and the urban-rural difference was not statistically significant). In contrast with changes in family structure, changes in family function are not obvious. While family structure has changed, family function appears to have remained relatively stable, raising two questions: Why has family function not changed along with family structure? Will a change to family structure lead to a change in family function? An example of a pathological phenomenon may help us analyze this problem. 
Table 3 General linear regression for the effect of family structure and function on mental health (GHQ-12)

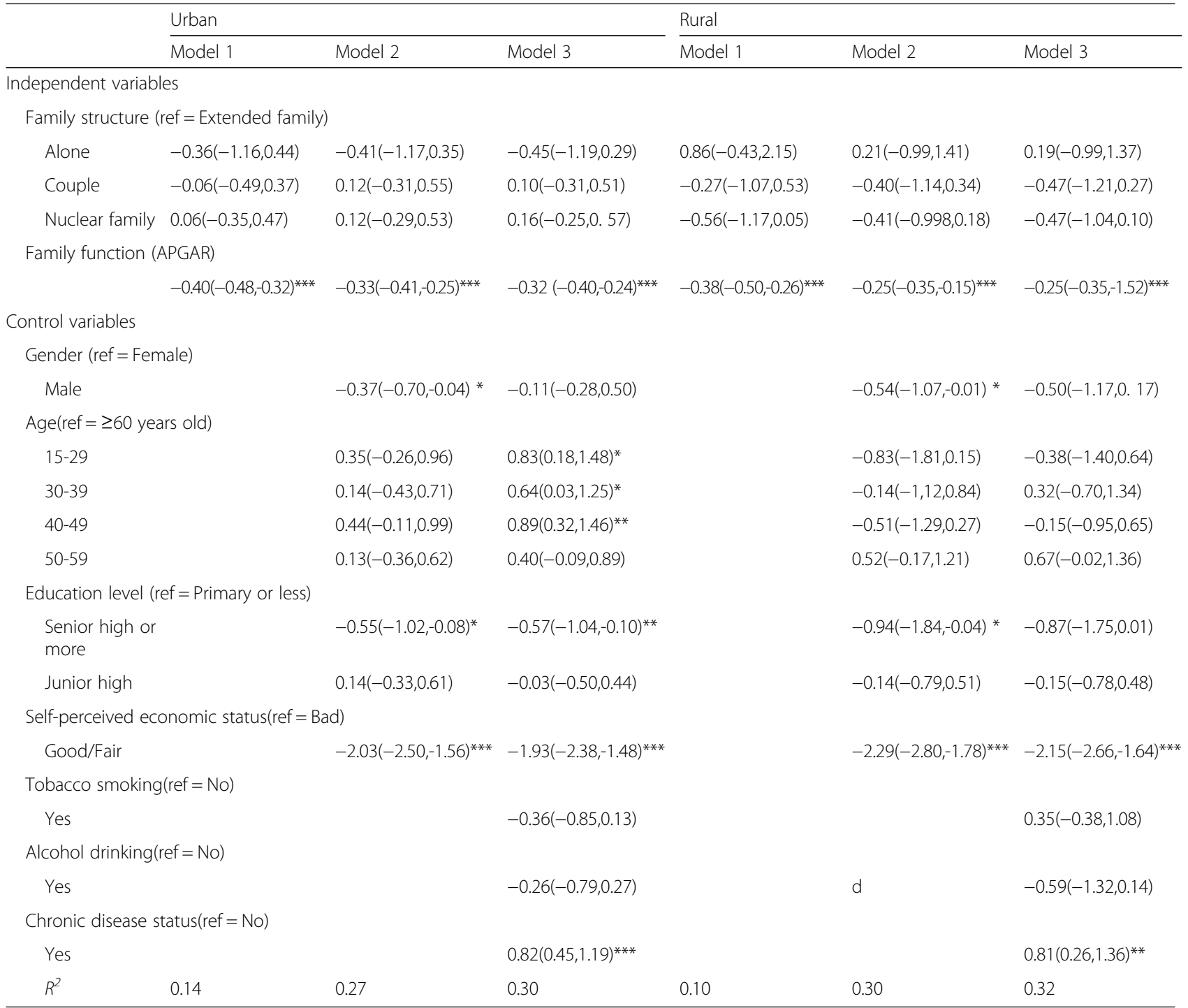

Note: Model 1 only use family structure and function; then Model 2, age, gender, education level, self-perceived economic status were added; then Model 3, tobacco smoking, alcohol drinking and chronic disease status were added

$p<0.05^{*}, p<0.01^{* *}, p<0.001^{* * *}$

Cirrhosis of the liver can be caused by a virus, but the liver may still function well (because of physiological compensation) when the disease is in its early stages or is relatively mild. Similarly, the current changes in family structure may be at an early stage, and so family function may still be good. Long-term changes in family structure could lead to changes in family function [33], so we need to take measures as early as possible to ensure that healthy functioning continues. This is very important during the transition period, especially for developing countries that are currently at a disadvantage in global competition.

\section{Mental health}

This study showed that the overall proportion of mental disorders is relatively high, and higher for those in rural areas than urban areas $(46.89 \%$ vs $26.92 \%)$. This result reveals the severity and breadth of mental health with social change, especially for rural residents [34-38]. One reason for this result may be the economic conditions in urban and rural areas [39]. The percentage of self-reported economic status deemed "bad" among urban and rural residents is 14.89 and $35.79 \%$, respectively. Poor economic conditions will increase burdens not only on living conditions and social status, but also on other factors including health and children's education. A further reason for this result is the reduction of the rural population and the growth of the urban-rural gap. Combined with the rise of egoism and money worship, this could result in greater mental ill health. 


\section{The effect of family structure and function on mental health}

Our multivariate analysis showed that better family functioning was associated with better mental status. Previous research on special populations such as children, adolescents, and immigrants showed that family function had a protective effect on mental health [8-10, 40]. The present study confined such protection effects to the adult population. It is worth noting that the impact of family structure on mental health, whether urban or rural, was not statistically significant. When looking at the combined effects of family structure and function, the external form of family (family structure) may not be important, while the internal qualify of role (family function) might be key.

With irreversible globalization, long-term changes in family structure may affect family function, thereby affecting the mental health of family members. Whether traditional culture or social reality, China's traditional family functions cannot be replaced. Chinese families not only provide the functions of production, education, maternity, and pension, but also that of psychological comfort and support, which involves the health of all family members, as well as the stability and development of society as a whole. Faced with social change and health problems, individuals and families may be powerless. Thus, all facets of society should work together, which is particularly important for developing countries struggling to advance under globalization.

\section{Limitations}

First, the response rate in this study was relatively low, which could produce selection bias. With regard to the employment status of the adult population, our door-todoor survey in urban areas was conducted during weekends (Saturday and Sunday), rather than during the week (Monday to Friday) when adult participants were more likely to be out at work. However, because of the residents' reluctance to participate in the survey, especially in urban areas, it was very difficult for us to improve the response rate. Moreover, large families are more likely to left family member stay at home, and residents' reluctance to participate from same families might be relatively lower, so their participation rate would be high. Low response rates as well as the corresponding selection bias may be a reflection of social change on family structure and function. Second, as mentioned above, we were unable to determine the proportion of urban residents that had originated from rural areas in the past 10-20 years, which would affect the depth of the current study. Third, our sample was confined to three cities in a central province in China, which may reduce the generalizability of our findings. Forth, this study was a cross-sectional design, meaning that the statistical analysis neglects the term of time in the inferences.
Thus, the observed associations could not be considered causal, and there is a high possibility that these associations are false positives. The findings should be examined and replicated in a study with prospective design to examine the associations. Also, the observed effect of family structure and function on mental health could be caused by residual confounding. Additional, we applied linear models to examine this association taking into account some factors (age, gender, education, marital status, and family economic status), other factors not considered might act as confounders in this study.

\section{Conclusion}

As the world's largest developing country, the family structure and function as well as their impact on mental health are particularly noteworthy during China's transition. Combined the effects of family structure and function, the external form of family (family structure) may not be important, the internal quality of role (family function) might be the key. Improving the residents' family function would be a priority strategy for family practice with their mental health.

\section{Abbreviations}

APGAR: Adaptation Partnership Growth Affection Resolve; GHQ-12: the 12item General Health Questionnaire

\section{Acknowledgements}

The authors would like to thank all of participates for their great support which made this study possible.

\section{Funding \\ This study was supported by grants from Health Policy Research Fund of Health Bureau of Hubei Province, China. (№ ZC2010-8). The funders had no role in study design, data collection and analysis, decision to publish, or preparation of the manuscript.}

\section{Availability of data and materials}

The study data are available to other researchers upon request for the corresponding author.

\section{Authors' contributions}

YC and LZ have contributed equally to this work. YC FW LZ PZ BY and YL have conceived and designed the experiments. YC FW LZ PZ and BY have performed the experiments. YC and FW have analyzed the data. YC, LZ and $Y L$ have written the paper. All authors have read and agree to publish the final manuscript.

\section{Competing interests}

The authors declare that they have no competing interests.

Consent for publication

Not applicable.

Ethics approval and consent to participate

This study was approved by the ethics committee of the Ethics Committee of Tongji Medical College, Huazhong University of Science and Technology [2010] 14 , and was therefore performed in compliance with the Helsinki Declaration of 1964. All participants were informed of the purpose of our study and signed an informed consent form before participating in the survey.

\section{Publisher's Note}

Springer Nature remains neutral with regard to jurisdictional claims in published maps and institutional affiliations. 
Received: 24 February 2016 Accepted: 14 April 2017

\section{Published online: 05 May 2017}

\section{References}

1. Zhang M, Rasiah R. Globalization, industrialization and labour markets in China. J Asia Pac Econ. 2015;20:14-41.

2. Zhou F. Social impact of family structure and policy recommendations. Acad J Zhongzhou (in Chinese). 2014;9:83-6.

3. Chen J, Chen S, Landry PF. Urbanization and mental health in China: linking the 2010 population census with a cross-sectional survey. Int J Environ Res Public Health. 2015;12:9012-24

4. Feng D, Ji L, Xu L. Effect of subjective economic status on psychological distress among farmers and non-farmers of rural China. Aust I Rural Health. 2015;23:215-20.

5. Jiang Q, Sanchez-Barricarte J. The 4-2-1 family structure in China: a survival analysis based on life tables. Eur J Ageing. 2011;8:119-27.

6. Cooper CE, McLanahan SS, Meadows SO, Brooks-Gunn J. Family structure transitions and maternal parenting stress. J Marriage Fam. 2009;71(3):558-74.

7. Yi Z. Changes in family structure in China: A simulation study. Popul Dev Review. 1986;12(4):675-703.

8. Wu Q, Chow J. Social service utilization, sense of community, family functioning and the mental health of new immigrant women in Hong Kong. Int J Environ Res Public Health. 2013;10:1735-46.

9. Yen CF, Yang P, Wu YY, Cheng CP. The relation between family adversity and social anxiety among adolescents in Taiwan: effects of family function and self-esteem. J Nerv Ment Dis. 2013;201(11):964-70.

10. Gau SF, Chou MC, Chiang HL, Lee JC, Wong CC, Chou WJ, Wu YY. Parental adjustment, marital relationship, and family function in families of children with autism. Res Autism Spectr Disord. 2012;6(1):263-70.

11. Blum RW, Beuhring T, Shew ML, Bearinger LH, Sieving RE, Resnick MD. The effects of race/ethnicity, income, and family structure on adolescent risk behaviors. Am J Public Health. 2000;90(12):1879-84.

12. Rodríguez-Sánchez E, Pérez-Peñaranda A, Losada-Baltar A, PérezArechaederra D, Gómez-Marcos MÁ, Patino-Alonso MC, García-Ortiz L. Relationships between quality of life and family function in caregiver. BMC Fam Pract. 2011;12:19.

13. Evans-Lacko S, Knapp M, McCrone P, Thornicroft G, Mojtabai R. The mental health consequences of the recession: economic hardship and employment of people with mental health problems in 27 European countries. PLoS ONE. 2013;8:e69792.

14. Bhugra D, Mastrogianni A. Globalisation and mental disorders - Overview with relation to depression. Br J Psychiatry. 2004;184:10-20.

15. Colton T, Janzen B, Laverty W. Family structure, social capital, and mental health disparities among Canadian mothers. Public Health. 2015;129(6):63947.

16. Zeng Y, Li W, Liang ZW. Current situation, regional differences and trends of Chinese family structure. Popul Sci China (in Chinese). 1992;2:1-12.

17. Smilkstein G, Ashworth C, Montano D. Validity and reliability of the family APGAR as a test of family function. J Fam Pract. 1982;15(2):303-11.

18. LV F, Gu Y. The family APGAR scale and its clinical application. Foreign Med Sci (Series of Hospital) Manage (in Chinese). 1995;2:56-9.

19. Nan H, Lee PH, Ni MY, Chan BH, Lam TH. Effects of depressive symptoms and family satisfaction on health related quality of life: the Hong Kong FAMILY study. PLoS ONE. 2013;8(3):e58436.

20. Kim YS, Lee MJ, Suh YS, Kim DH. Relationship between family meals and depressive symptoms in children. Korean J Fam Med. 2013;34(3):206-12.

21. Caldwell C, Horne A, Davidson B, Quinn W. Effectiveness of a multiple family group intervention for juvenile first offenders in reducing parent stress. J Child Fam Stud. 2007;16(3):443-59.

22. Goldberg DP, Blackwell B. Psychiatric illness in general practice: a detailed study using a new method of case identification. Br Med J. 1970;1(5707): 439-43.

23. Donath $\mathrm{S}$. The validity of the 12-item General Health Questionnaire in Australia: a comparison between three scoring methods. Aust N Z J Psychiatry. 2001;35(2):231-5.

24. Gao F, Luo N, Thumboo J, Fones C, Li SC, Cheung YB. Does the 12-item General Health Questionnaire contain multiple factors and do we need them? Health Qual Life Outcomes. 2004;2:63.

25. Bell T, Watson M, Sharp D, Lyons I, Lewis G. Factors associated with being a false positive on the General Health Questionnaire. Soc Psychiatry Psychiatr Epidemiol. 2005;40(5):402-7.
26. Goldberg DP, Gater R, Sartorius N, Ustun TB, Piccinelli M, Gureje O, Rutter C. The validity of two versions of the GHQ in the WHO study of mental illness in general health care. Psychol Med. 1997;27(1):191-7.

27. Wang $W$, Ding $L$, Liao $Z$. The best thresholds and the screening features among the three scoring methods of the 12-item General Health Questionnaire. Chin J Psychiatry. 2012;45:349-53.

28. Chen RS, Ye C, Cai YL, Xing XS, Chen Q. The impact of rural out-migration on land use transition in China: Past, present and trend. Land Use Policy. 2014:40:101-10.

29. Yang JH, He ZH. Continuity or change? Chinese family in transitional era. Popul Res (in Chinese). 2014;38(2):36-51.

30. Zhou SH, Xie M, Kwan MP. Ageing in place and ageing with migration in the transitional context of urban China: A case study of ageing communities in Guangzhou. Habitat Int. 2015;49:177-86.

31. Qi XA. Study on the influence and tendency of the marriage and family under the social culture vicissitude. Popul J (in Chinese). 2009;3:31-6.

32. Bhat MA, Rather TA. Socio-economic factors and mental health of young people in india and China: an elusive link with globalization. Asian Soc Work Pol Rev. 2012;6:1-22

33. Jung JA. Changing Chinese familial structure and its effects on family's financial support for the elderly: future implications for family value in China. Korean J Area Stud. 2013;31:81-107.

34. Liu J, Ma H, He YL, Xie B, Xu YF, Tang HY, Li M, Hao W, Wang XD, Zhang MY. Mental health system in China: history, recent service reform and future challenges. World Psychiatry. 2011;10:210-6.

35. Phillips MR, Zhang J, Shi Q, Song Z, Ding Z, Pang S, Li X, Zhang Y, Wang Z. Prevalence, treatment and associated disability of mental disorders in four provinces in China during 2001-05: an epidemiological survey. Lancet. 2009: 2041-2053:373.

36. Keqing L, Ze C, Lijun C, Qinpu J, Guang S, Haoran W, Jing H, Wuwen Z, Jianguo $X$, Yanping Z. Epidemiological survey of mental disorders in the people aged 18 and older in Hebei Province. Asian J Psychiatr. 2008;1:51-5.

37. Chen SL, Ni XL. The rural citizen's psychological status and investigation approach. Chin Med Ethics (in Chinese). 2006;19:117-8.

38. Wang S, Li B, Ungvari GS, Ng CH, Chiu HF, Kou C, Liu Y, Tao Y, Wu Y, Fu Y, Qi Y, Yu Y, Xiang YT. Poor mental health status and its associations with demographic characteristics and chronic diseases in Chinese elderly. Soc Psychiatry Psychiatr Epidemiol. 2016. [Epub ahead of print].

39. Andrews AR, Jobe-Shields L, Lopez CM, Metzger IW, de Arellano MAR, Saunders B, Kilpatrick DG. Polyvictimization, income, and ethnic differences in trauma-related mental health during adolescence. Soc Psychiatry Psychiatric Epidemiol. 2015;50(8):1223-34.

40. Zito RC. Family Structure History and Teenage Cohabitation: Instability, Socioeconomic Disadvantage, or Transmission? J Fam Issues. 2015;36(3): 299-325.

\section{Submit your next manuscript to BioMed Central and we will help you at every step:}

- We accept pre-submission inquiries

- Our selector tool helps you to find the most relevant journal

- We provide round the clock customer support

- Convenient online submission

- Thorough peer review

- Inclusion in PubMed and all major indexing services

- Maximum visibility for your research

Submit your manuscript at www.biomedcentral.com/submit
Biomed Central 